\title{
Bending Characteristics of Fiber Long-Period Gratings With Cladding Index Modified by Femtosecond Laser
}

\author{
T. Allsop, M. Dubov, A. Martinez, F. Floreani, I. Khrushchev, D. J. Webb, and I. Bennion
}

\begin{abstract}
A femtosecond laser has been used to asymmetrically modify the cladding of fiber containing long-period gratings. Following modification, devices in single-mode fiber are shown to be capable of sensing the magnitude and direction of bending in one plane by producing blue and red wavelength shifts depending upon the orientation of the bend. The resulting curvature sensitivities were -1.62 and $+3.82 \mathrm{~nm} \cdot \mathrm{m}$. Devices have also been produced using an elliptical core fiber to study the effects of the cladding modification on the two polarization eigenstates. A cladding modification applied on the fast axis of the fiber is shown to affect the light in the fast axis much more significantly than the light in the orthogonal state; this behavior may ultimately lead to a sensor capable of detecting the direction of bending in two dimensions for applications in shape sensing.
\end{abstract}

Index Terms-Curvature measurement, long-period fiber gratings, polarization dependence.

\section{INTRODUCTION}

A long-period grating (LPG) is an axially periodic refractive-index variation in the core of a single-mode optical fiber that couples light in the core to fiber cladding modes at discrete wavelengths [1]; LPGs have been suggested for many potential applications in the field of sensing [2]. A promising application of fiber LPG devices is as a curvature sensor [3], but a problem here is that in a symmetric fiber, the direction of the bend cannot be determined, and thus, at present, LPGs written in standard fiber cannot be used as true orientation sensors. Some limited success in making orientation sensors has been achieved with LPGs written in asymmetric fibers such as D-shaped cladding fiber [4] or eccentric core fiber [5]. Although the resulting devices can determine the orientation of the bend, they still present some practical problems; for example, the cost of the special fiber and the problem of splicing to standard optical fiber. These approaches can also lead to problems with polarization dependence [5], [6] due to the birefringence/asymmetry of the fiber. This adds to the complexity of the fabrication procedure and interrogation of the sensors, which raises the system cost.

We report on the spectral behavior of LPGs as a function of curvature following modification of the cladding using an $800-\mathrm{nm}$ femtosecond laser in two types of optical fiber. The

Manuscript received August 10, 2005; revised April 4, 2006.

The authors are with the Photonics Research Group, Aston University, B4 7ET Birmingham, U.K. (e-mail: t.d.p.allsop@astons.ac.uk; m.dubov@aston. ac.uk; martinea@aston.ac.uk; floreanf@imap.aston.ac.uk; i.khrushchev@ aston.ac.uk; d.j.webb@aston.ac.uk; I.Bennion@aston.ac.uk).

Digital Object Identifier 10.1109/JLT.2006.878037 types of fibers used were a conventional step-index telecommunication fiber [single-mode fiber (SMF)] and a step-index elliptical core (E-core) fiber (a polarization-maintaining fiber with one of the simplest nonradially symmetric fiber geometries). The reason for choosing the two fibers was, first, to investigate the spectral sensitivity to curvature and, second, to study the polarization properties of the LPG devices, which is a major consideration when using asymmetric fiber for bend sensors [5], [6].

\section{FABRICATION}

The novel sensing devices were fabricated in two stages, namely 1) conventional ultraviolet (UV) LPG inscription and 2) introduction of an asymmetric change in the cladding's refractive index using the femtosecond laser. The standard circularly symmetric SMF had core and cladding radii of 3.5 and $62.5 \mu \mathrm{m}$, respectively. For comparison, LPGs were also fabricated in the E-core fiber that had a major semiaxis of $5 \mu \mathrm{m}$ and a minor semiaxis of $3 \mu \mathrm{m}$ with a cladding radius of $62.5 \mu \mathrm{m}$. None of the fibers was specifically designed to be photosensitive, and hence, the photosensitivity was increased by hydrogenation at a pressure of 120 bar for a period of two weeks at room temperature.

The LPGs were inscribed into the fibers using a frequencydoubled argon ion laser with a point-by-point writing technique. The chosen grating period for the SMF was $400 \mu \mathrm{m}$ with a length of $5 \mathrm{~cm}$, whereas for the E-core fiber, values of 250 and $400 \mu \mathrm{m}$ were used, each with a grating length of $5 \mathrm{~cm}$.

Asymmetric changes in the refractive index of the cladding of the fiber were introduced by a near-infrared (NIR) femtosecond laser $(800 \mathrm{~nm}$ Spitfire/Evolution, Titanium/Sapphire system, Spectra-Physics Lasers). The femtosecond laser radiation was focused at a predetermined point near the core with a 100 times $(N A=0.55)$ microscopic objective, whereas the fiber was moved in a direction parallel to the fiber axis. The translation speed was $100 \mu \mathrm{m} / \mathrm{s}$. A cylindrical lens $(f=100 \mathrm{~mm})$ was put in the proximity of the microscopic objective to shape the laser beam at the point of inscription. The femtosecond laser system produced a $1-\mathrm{kHz}$ train of $150-\mathrm{fs}$ pulses at $800 \mathrm{~nm}$, and the pulse energy was approximately $1 \mu \mathrm{J}$ after attenuation (see Fig. 1). Other details of the experimental setup can be found elsewhere [6].

Two SMF LPGs (period $400 \mu \mathrm{m}$ and length $5 \mathrm{~cm}$ ) were modified by the femtosecond laser radiation. First, in LPG1, three continuous waveguides were written in the same plane and 


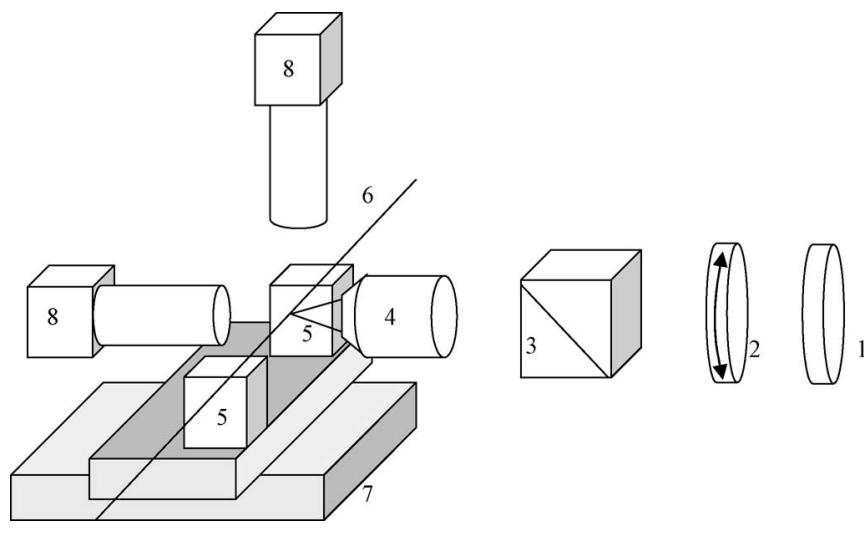

Fig. 1. Optical layout of inscription scheme. The light passes a shutter (1), a half-wave plate (2), Glan prism (3), $100 \times$ long working distance microscopic objective (4), and is then focused in the fiber core (6). Two three-dimensional (3-D) translation stages for alignment are mounted on top of the high-precision computer-controlled two-dimensional (2-D) stage (7). We use two chargecoupled device (CCD) cameras (8) with optical zooming system for alignment and on-line monitoring of the inscription process.

parallel to the core in the region of the LPG. From the previous paper, we estimate the induced refractive index increase to be approximately $1.5 \times 10^{-3}$ [6]. The intensity of the laser was kept at a level to make single spots overlap, thus making the waveguides quasi-continuous, with a visual diameter of approximately $5 \mu \mathrm{m}$. Two of the waveguides were placed $2 \mu \mathrm{m}$ from either side of the core, and the third waveguide was placed $20 \mu \mathrm{m}$ from the core in the same plane as the other waveguides. The diameter of each waveguide was approximately $5 \mu \mathrm{m}$. Second, in LPG2, a single continuous waveguide was written parallel to the core at a distance of $5 \mu \mathrm{m}$ from the core. See Figs. 2 and 3 for the geometry of the device and an example microscopic image.

Two E-core fibers were also modified with the same procedure outlined above. First, the two polarization axes of the fiber were identified and labeled for orientation; this was achieved by illuminating one end of the E-core fiber by butt-coupling it to a polarized light source and connecting the other end to a polarimeter. The illuminated end of the fiber was rotated so that only one polarization state of the fiber was excited. The fiber was then tagged, the labels on the fiber denoting one polarization state with the other at $90^{\circ}$ to the label. In the case of LPGE1 (period $400 \mu \mathrm{m}$ and length $5 \mathrm{~cm}$ ), a single continuous waveguide was written parallel to the slow axis of the core at a distance of $\sim 20 \mu \mathrm{m}$ from the core, and a second continuous waveguide was written parallel to the first but rotated $90^{\circ}$ about the fiber axis, again, at a distance of $\sim 20 \mu \mathrm{m}$ from the core. Second, in LPGE2 (period $250 \mu \mathrm{m}$ and length $5 \mathrm{~cm}$ ), a single continuous waveguide was written parallel to the fast axis of the core at a distance of $20 \mu \mathrm{m}$ from the core (see Figs. 2 and 3 ).

The radial distances for the femtosecond-laser-inscribed index modifications were chosen in an attempt to maximize the dependence of the cladding modes' spectral behavior on curvature. The distance was selected by finding the radial maxima for the electric field of the cladding modes associated with the observed attenuation bands. The electric field radial profile was calculated using the approach given in [1].
The characterization of the attenuation bands was carried out by illuminating the LPGs using a broadband light source and observing the transmission spectrum with an optical spectrum analyzer (OSA) with an accuracy of $0.05 \mathrm{~nm}$. Furthermore, for the E-core fiber, a polarizer and a polarization controller were used in conjunction with the broadband light source. The experiment revealed an approximately 21-nm separation between the attenuation bands associated with the two orthogonal polarization states for the E-core fiber for an LPG having a period of $400 \mu \mathrm{m}$ and a length of $5 \mathrm{~cm}$ (LPGE1) (see Fig. 4).

Following the femtosecond modification of the E-core fiber, two observations were made. First, that both blue and red wavelength shifts of both polarization states were measured. For the $p$-polarization state, there were shifts of -0.38 to $+1.90 \mathrm{~nm}$, which are equivalent to changes in index ranging from $-4.1 \times 10^{-6}$ to $+2.0 \times 10^{-5}$, assuming that the core index is unchanged [7]. For the $s$-polarization state, the shifts ranged from -1.66 to $+1.50 \mathrm{~nm}$, which are equivalent to changes in refractive index from $-1.5 \times 10^{-5}$ to $+1.6 \times 10^{-5}$. These index changes are dependent upon the particular cladding mode under consideration due to the spatial variation of the individual cladding modes and their consequently differing overlaps with the femtosecond laser index perturbation. Second, it was observed that the separation between the attenuation bands corresponding to the same cladding mode but observed using the light in the two fiber eigenaxes had reduced by approximately $0.4 \mathrm{~nm}$ (see Fig. 5), which shows an equivalent change in the birefringence of $\Delta n \sim 1.7 \times 10^{-6}$ [7]. A possible reason for this is that one of the two inscribed waveguides might have led to a greater change in the refractive index than the other. Evidence is provided by Fig. 3(b), where a difference between the two waveguides is visible.

The femtosecond laser inscription introduces an asymmetric variation of index in the cladding of the SMF, which changes the polarization properties of the fiber grating devices. In an ideal circularly symmetric fiber, the fundamental core and cladding modes have two orthogonal polarization states, i.e., $H E_{1, n}^{x}$ and $H E_{1, n}^{y}$, but because of symmetry, both sets of modes are degenerate. However, when the refractive-index profile of the core or cladding is not azimuthally symmetric, the degeneracy is broken. The asymmetry gives a geometric birefringence in the core/cladding modes [7], [8]. Therefore, the propagation constants or the effective indexes of the two polarization modes become different. Inspecting the polarization properties of the LPGs in SMF after the inscription of the cladding modification shows that on top of this splitting of the polarization states, there is an overall red shift in all attenuation bands of $\sim 0.6 \mathrm{~nm}$, giving an equivalent change in the effective index of $\sim 2.6 \times 10^{-6}$.

\section{Spectral Bending Characteristics}

The LPG devices were inserted into a 4-mm-diameter flexible rubber tube (ensuring that the fiber did not flip to another orientation), and both the fiber and the rubber tube were attached to two rotational clamps and suspended between two towers with one of the clamps being mounted on a translation stage. This was moved inward to induce a bend in the rubber 


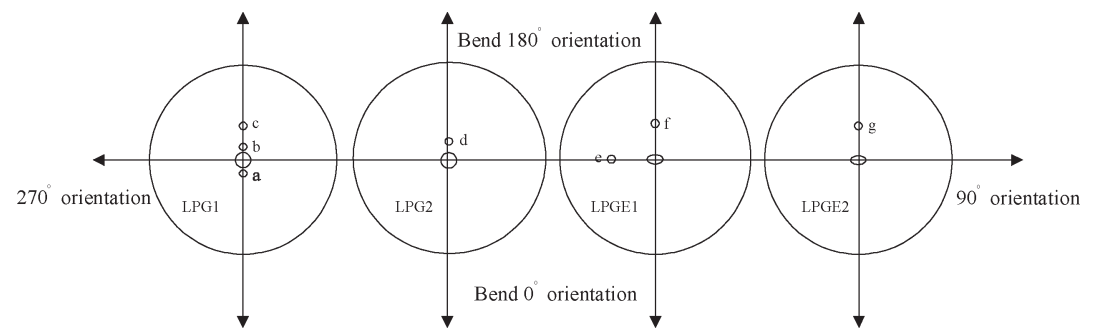

Fig. 2. Cross-sectional view of the cladding modifications. The bend orientation indicates the direction of the inside of the bend. Fiber devices LPG1 and LPG2 are SMFs with modifications: (a) $2 \mu \mathrm{m}$; (b) $2 \mu \mathrm{m}$; (c) $20 \mu \mathrm{m}$; and (d) $5 \mu \mathrm{m}$. Fiber devices LPGE1 and LPGE2 are E-core fibers with modifications: (e) $20 \mu \mathrm{m}$; (f) $20 \mu \mathrm{m}$; and (g) $20 \mu \mathrm{m}$. All distances are measured from the center of the fiber.
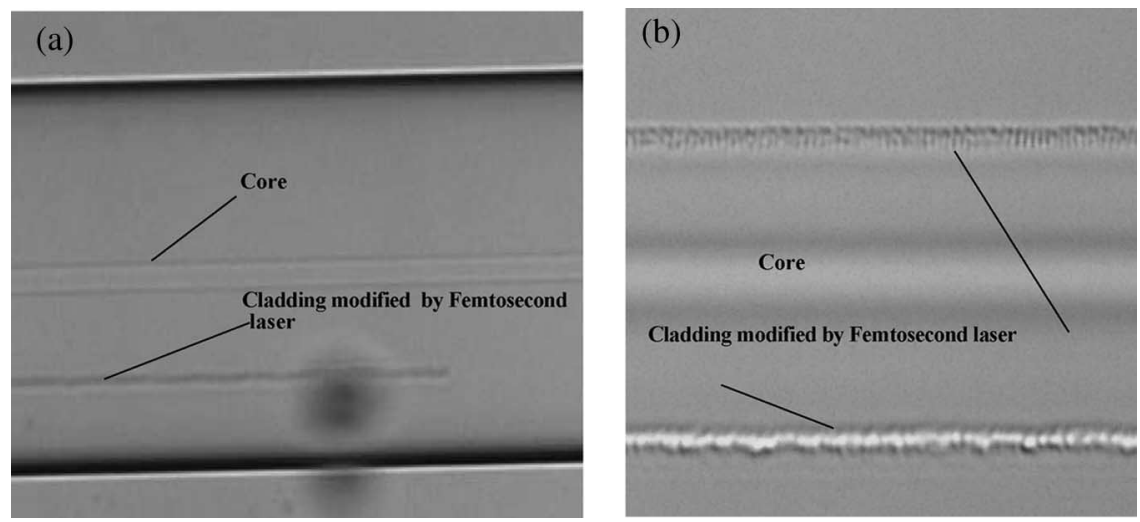

Fig. 3. Examples of the optical fiber cladding modified by a femtosecond laser. (a) Single line inscribed in SMF. (b) Two lines inscribed in the E-core fiber.
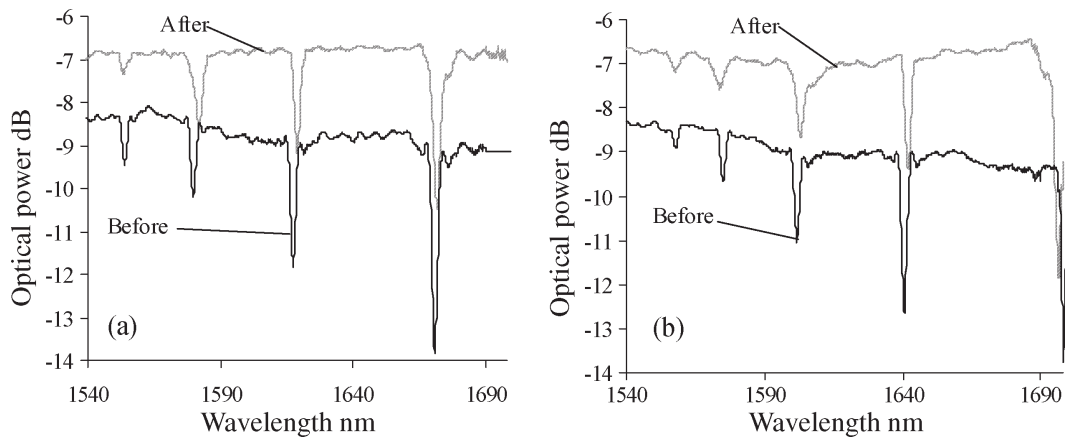

Fig. 4. Typical changes in the wavelength of the attenuation bands of LPGE1 (period $400 \mu \mathrm{m}$ and length $5 \mathrm{~cm}$ ) for the two polarization states due to cladding modification by the femtosecond laser. (a) $p$-polarization state. (b) $s$-polarization state.

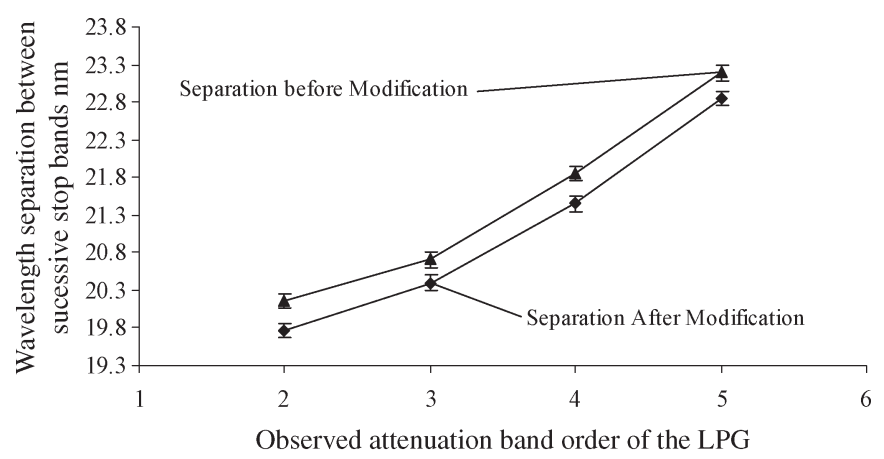

Fig. 5. Variation in the spectral separation between the orthogonal polarization states of the same cladding mode order before and after the femtosecond laser cladding modification.

tube and, hence, the optical fiber (see Fig. 6). Prior to bending, the fiber could be rotated axially so that the bending could be applied at various angles to the axis defined by the core

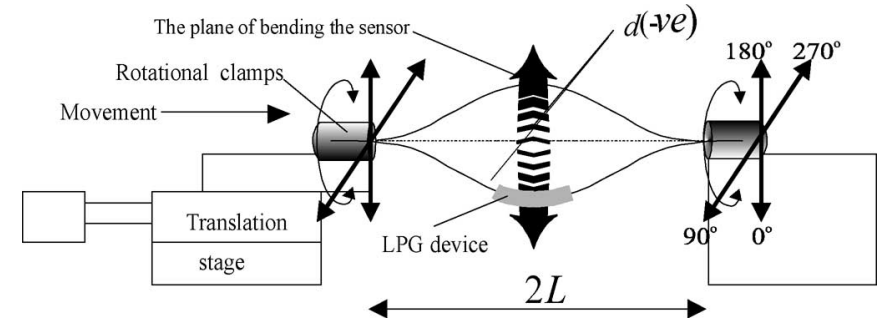

Fig. 6. Schematic of the bending/rotational test rig.

and the cladding modifications. The axes defining the $0^{\circ}$ axis are indicated in Figs. 2 and 6. The bend sensitivities of these LPGs were investigated using a broadband light source and a polarizer, which, in turn, was connected to a polarization controller; the light from this arrangement illuminated the LPGs, and observations of the transmitted spectra were made using an OSA. The polarization controller was used to maximize the 

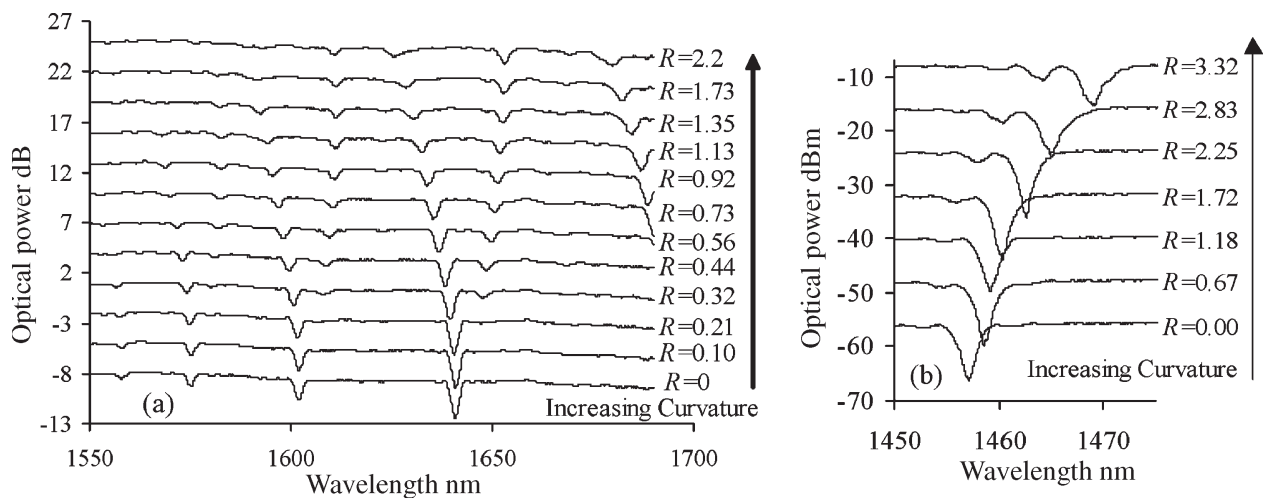

Fig. 7. (a) Transmission spectra of an LPG in the E-core fiber with no cladding modification showing attenuation bands associated with the fast axis (LPGE2, period $250 \mu \mathrm{m}$, length $4 \mathrm{~cm}$ ) with increasing curvature from 0 to $2.2 \mathrm{~m}^{-1}$. (b) Typical transmission spectrum of an LPG written into the SMF optical fiber for comparison (period $240 \mu \mathrm{m}$, length $5 \mathrm{~cm}$ ). Traces are offset vertically for clarity.

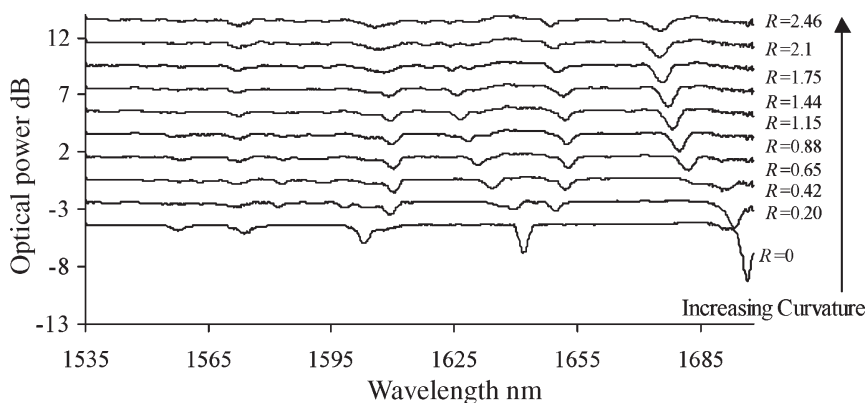

Fig. 8. Transmission spectra of an LPG in the E-core fiber following cladding modification showing attenuation bands associated with the fast axis (LPGE2, period $250 \mu \mathrm{m}$, length $4 \mathrm{~cm}$ ) with increasing curvature from 0 to $2.46 \mathrm{~m}^{-1}$. Traces are offset vertically for clarity.

light in one polarization state so that one state could be studied at a time.

The sensor's curvature $R$ is given by [9]

$$
R=\frac{2 \cdot d}{\left(d^{2}+L^{2}\right)}
$$

where $L$ is the half-distance between the edges of the two towers, and $d$ is the bending displacement at the center of the LPG. In Figs. 7 and 8, the experimental results are shown for LPGE2 with a period of $250 \mu \mathrm{m}$, the spectral behavior of this LPG being typical of all the LPGs written in the E-core fiber. The curvatures imposed on the LPG in the $0^{\circ}$ position are shown; the other positions were also investigated but only small variations in response were found. Both polarization states of the LPG were investigated with the results for the fast axis being shown in Fig. 7 (before cladding modification) and Fig. 8 (after cladding modification).

The details of the bend characteristics of the LPGs written into the E-core fiber without cladding modification are reported elsewhere [10], but briefly; by inspecting Fig. 7, we can see that as the LPG is subjected to increasing curvature, the "normal" attenuation bands experience a blue wavelength shift, but there is also the generation of "bend-induced" attenuation bands that have a red wavelength shift. Comparing these results to the standard SMF [Fig. 7(b)], two differences are observed: 1) For the SMF, a significant induced birefringence occurs at a curvature of approximately $2 \mathrm{~m}^{-1}$, beyond which, splitting of the

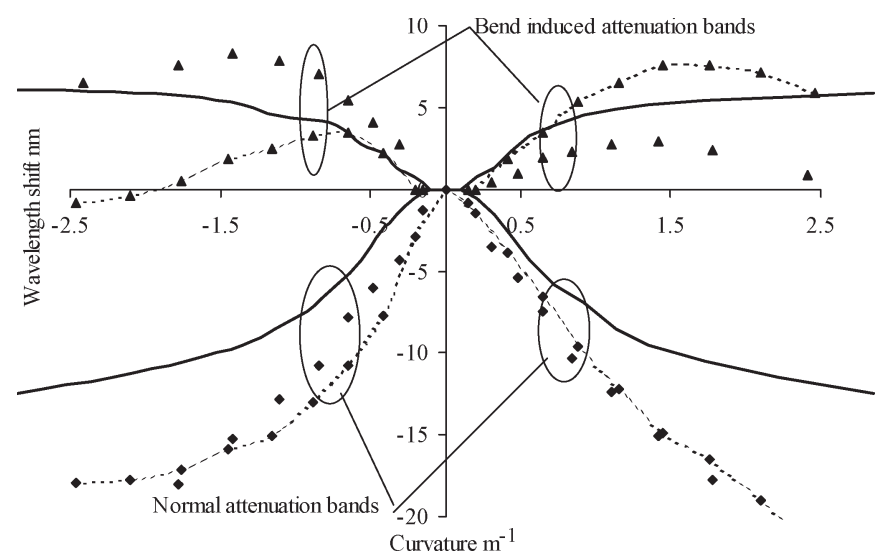

Fig. 9. Wavelength shift due to the curvature of the E-core fiber LPG attenuation bands at $1640 \mathrm{~nm}$ associated with the fast axis (LPGE2, period $250 \mu \mathrm{m}$, length $4 \mathrm{~cm})$ before cladding modification $\left(0^{\circ}\right.$ and $\left.180^{\circ}\right)(-)$ and after cladding modification $\left(---, 0^{\circ}\right.$ is $+\mathrm{Ve}, 180^{\circ}$ is $-\mathrm{Ve}$ and,$--- 90^{\circ}$ is $+\mathrm{Ve}, 270^{\circ}$ is $\left.-\mathrm{Ve}\right)$.

attenuation bands is observed; in the case of the LPGs written in the E-core fiber, the bend-induced attenuation band started to be evident at a curvature of only approximately $0.1 \mathrm{~m}^{-1}$. This is likely due to the intrinsic birefringence of the E-core fiber, which does not exist in the SMF. 2) The evolution with respect to curvature differs from the SMF; with E-core fiber, the bend-induced attenuation bands appear already separated from the normal attenuation bands and not from the splitting of the normal attenuation bands, as they do with SMF.

After the cladding modification of LPGE2, the bending characterization was repeated. It can be seen in Fig. 8 that the spectral response of the LPG is significantly different from the initial LPG device. This is shown more clearly in a plot of wavelength shift as a function of curvature for the fast axis of the 1640-nm band (see Fig. 9).

Fig. 9 shows a dramatic change in the spectral characteristics of LPGE2 for the fast axis; a clearer view of the effects of the cladding modification can be seen by subtracting the wavelength shift of the LPG before the cladding modification from the wavelength shift after the femtosecond laser processing (see Figs. 10 and 11). In these figures, the spectral behavior of the bend-induced attenuation bands are plotted; the normal 


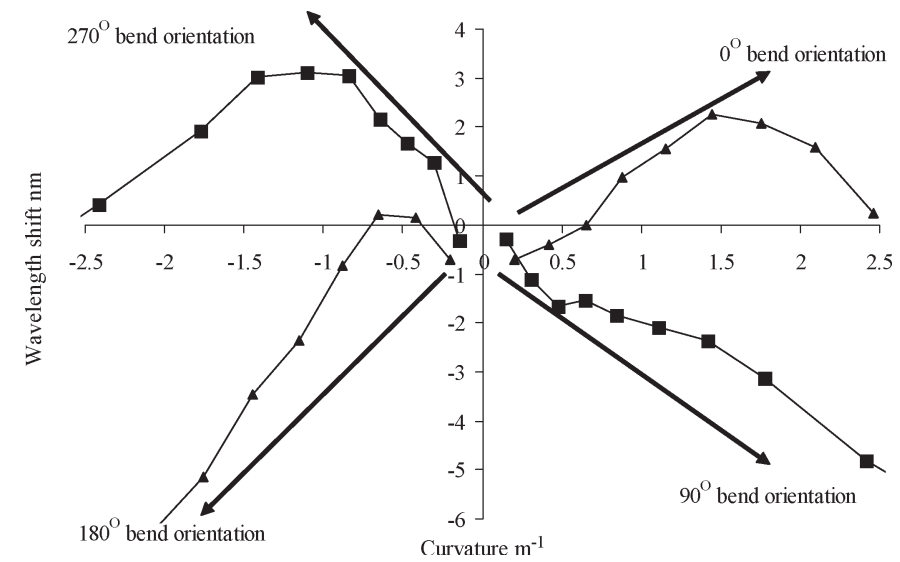

Fig. 10. Difference in the wavelength shifts of the fast axis of the 1640-nm bend-induced attenuation band (LPGE2, period $250 \mu \mathrm{m}$, length $4 \mathrm{~cm}$ ) before and after cladding modification.

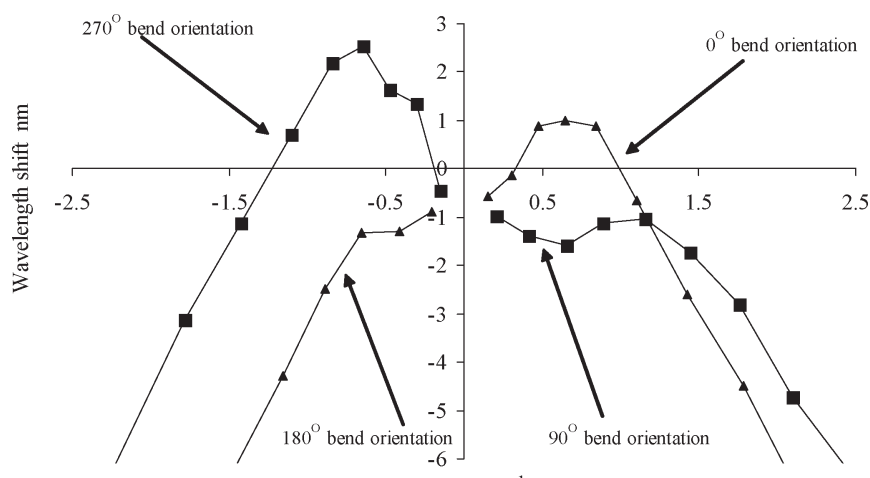

Curvature $\mathrm{m}^{-1}$

Fig. 11. Difference in the wavelength shifts of the slow axis of the 1640-nm bend-induced attenuation band (LPGE2, period $250 \mu \mathrm{m}$, length $4 \mathrm{~cm}$ ) before and after cladding modification.

attenuation bands showed less spectral alteration after the modification.

First, it was noticed that the spectral response of the normal attenuation bands to bending for the slow axis showed very little difference between the two directions of the bending, whereas the normal attenuation bands associated with the fast axis (shown in Fig. 9) do show noticeable differences in their response. The spectral sensitivities are expected to be different due to the fact that the light in the fast axis possesses an electric field that extends further into the cladding than the slow axis and thus will be affected more by the asymmetric modification of the cladding. In the same way, the light in the fast axis will also be affected more by the nonuniform strain profile induced by bending.

Second, inspection of the spectral response of the bendinduced attenuation bands shows major changes in the spectral response before and after modification for both slow and fast axes. The bend modifies the waveguide refractive-index profile, and the type of bend (i.e., concave or convex) has a significant effect on the overall refractive-index profile of the fiber due to the fiber asymmetry and thus affects the bend-induced asymmetric cladding modes more significantly. Inspecting the difference in the wavelength shift of the bend-induced attenuation bands for the fast axis before and after modification (Fig. 10) showed that the direction of the bend controlled to a significant degree the sign of the change in wavelength shift, with a generally increasing sensitivity for $0^{\circ}$ and $270^{\circ}$ and generally decreasing sensitivity at $180^{\circ}$ and $90^{\circ}$.

One aspect of these results deserves further comment; inspection of the geometry of the cladding modification and bend direction shown in Fig. 2 suggests that the effects of bending in the $90^{\circ}$ and $270^{\circ}$ directions should be the same due to the fiber symmetry. The fact that we do see a difference in the results for these two directions, we attribute to the difficulty of experimentally maintaining the alignment of the femtosecond beam with respect to the fiber core. We suspect that the cladding modification is not perfectly parallel to the core.

Inspecting the difference in wavelength shifts for the slow axis (Fig. 11) again shows significant change, but the wavelength changes are less easy to distinguish with bend orientation. This is probably due to the fact that the index modification was inscribed parallel to the fast axis of the core, and the two modes have differing spatial overlaps with the modified region. This suggests that with an appropriate index modification, this approach could be used to determine the bend orientation and, by using polarization dependence, produce a true vectorial fiber bend sensor.

Although the behavior of the attenuation bands of the E-core fiber is complex, there are similarities to the response of the SMF LPGs following cladding modification. Fig. 12 shows how the cladding modification affects the behavior of the bend-induced attenuation bands in LPG2. For LPG2, although the bend-induced attenuation band shows a dramatic change in its spectral response to curvature as a result of the cladding modification, the normal attenuation band close to $1500 \mathrm{~nm}$ showed very little change and is still unable to distinguish the bend direction. A summary of the bending characteristics for the normal attenuation band and the induced attenuation band before and after the femtosecond inscription is given in Table I for LPG2.

Examples of the transmission spectra of LPG1 for concave $\left(0^{\circ}\right)$ and convex $\left(180^{\circ}\right)$ bending are shown in Fig. 13.

First, the spectral sensitivity of the normal attenuation band was measured for both modes of bending, resulting in $d \lambda / d R=$ $+5.6 \pm 0.1 \mathrm{~nm} \cdot \mathrm{m}$ for concave bending and $d \lambda / d R=+4.6 \pm$ $0.2 \mathrm{~nm} \cdot \mathrm{m}$ for convex bending. Second, the bend-induced attenuation band was characterized and displayed a spectral sensitivity of $d \lambda / d R=-1.6 \pm 0.1 \mathrm{~nm} \cdot \mathrm{m}$ for concave bending and $d \lambda / d R=+3.8 \pm 0.5 \mathrm{~nm} \cdot \mathrm{m}$ for convex bending. The wavelength shifts as a function of curvature of the normal and the bend-induced attenuation bands of LPG1 are shown in Fig. 14.

As with LPG2, although the bend-induced attenuation band shows a dramatic change in its spectral response to curvature, the normal attenuation band at $1500 \mathrm{~nm}$ shows very little change and is thus still unable to distinguish between the two directions of bending. This behavior is similar to the spectral response of the slow axis normal attenuation bands of the modified E-core LPGs.

The polarization dependence of the curvature response of the modified SMF LPG was investigated by illuminating the sensor with nonpolarized and then polarized light. The maximum polarization dependence of the attenuation band is $\sim 0.6 \mathrm{~nm}$, 

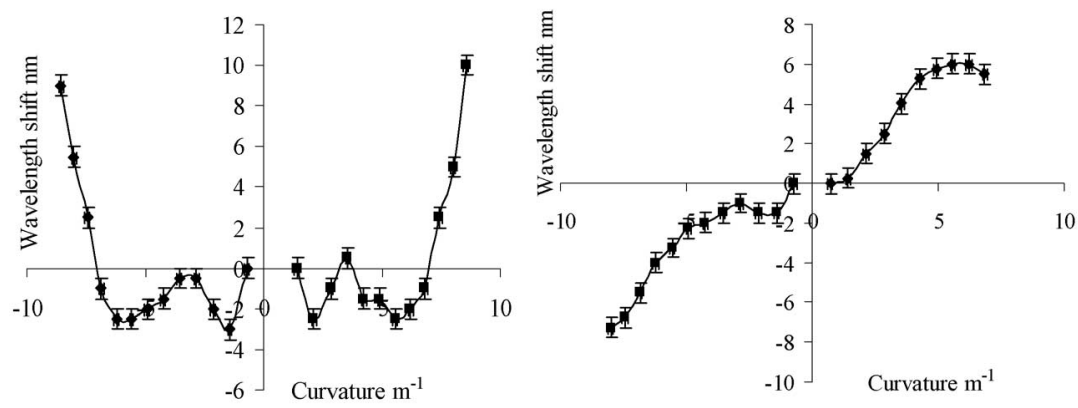

Fig. 12. Example of the spectral sensitivity of the induced attenuation band at $1610 \mathrm{~nm}$ before (left) and after (right) the femtosecond laser modification of LPG2.

TABLE I

SPectral Sensitivities to CuRvature For the Normal AtTEnuation BAND AND the Induced ATTENUATION BAND BEFORE AND AFTER FEMTOSECOND INSCRIPTION

\begin{tabular}{lllll}
\hline Spectral sensitivity, $\mathrm{nm} \cdot \mathrm{m}$ & \multicolumn{2}{c}{ Concave bending } & \multicolumn{2}{c}{ Convex bending } \\
& before & after & before & after \\
\hline Normal attenuation band, $1500 \mathrm{~nm}$ & $+3.2 \pm 0.3$ & $+2.8 \pm 0.3$ & $+2.3 \pm 0.2$ & $+1.6 \pm 0.3$ \\
Induced attenuation band, $1610 \mathrm{~nm}$ & $-0.23^{*}$ & $-0.91 \pm 0.04$ & $-0.30^{*}$ & $+1.01 \pm 0.05$ \\
* The assumption of linearity of the spectral sensitivity is very poor, but these figures are \\
to give a rough comparison for standard and modified bend sensors.
\end{tabular}
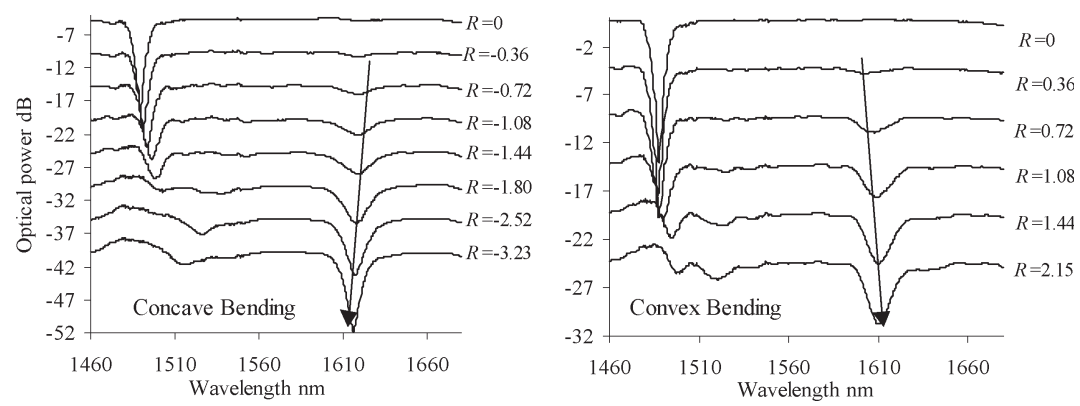

Fig. 13. Transmission spectra of LPG1 as a function of direction and amount of curvature. Traces are offset vertically for clarity.
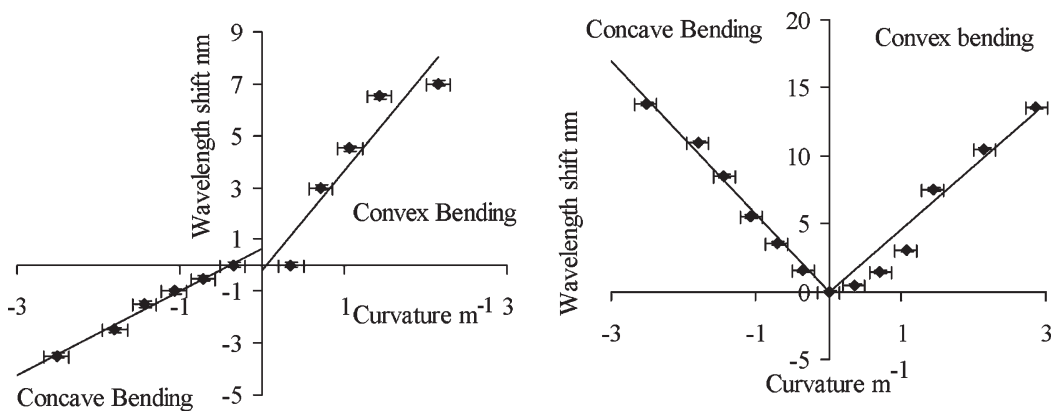

Fig. 14. Spectral sensitivity of the induced attenuation band (left) at $1610 \mathrm{~nm}$ and the normal attenuation band (right) at $1500 \mathrm{~nm}$ for LPG1, following modification.

which is low compared with the polarization dependence of the other previously reported bend sensors such as LPGs in E-core $\mathrm{D}$-shaped fibers, where the shift is approximately $15 \mathrm{~nm}$ [11].

The observed spectral behavior is explained by the introduction of the asymmetry as seen previously in the response to the bending of LPGs in D-shaped fiber [4]. LPG1 and LPGE2 were modeled using a finite-element package (FEMLAB) to obtain the effective refractive indexes of the core $n_{\text {co }}(\lambda)$ and the cladding modes $n_{\mathrm{cl}}(\lambda)$ at various wavelengths. The geometric specifications of these fibers are given in Section II with the exception of the refractive-index change in the cladding, which has been modified by the femtosecond laser, where the index change was adjusted to obtain a reasonable fit with experimental data. The center wavelength $\lambda_{\nu}$ of an attenuation band is specified by the following phase-matching condition [2]:

$$
\delta n_{\mathrm{eff}_{\nu}} \Lambda=\lambda_{\nu}
$$

where

$\delta n_{\mathrm{eff}_{\nu}}=\left[n_{\mathrm{co}}^{01}\left(\lambda, \varepsilon, T, n_{1}, n_{2}, R\right)-n_{\mathrm{cl}}^{1 \nu}\left(\lambda, \varepsilon, T, n_{1}, n_{2}, n_{s,}, R\right)\right]$ 

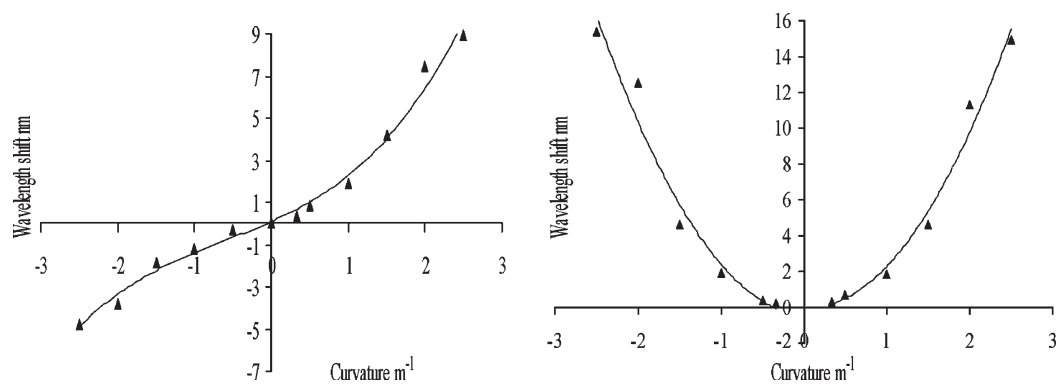

Fig. 15. Theoretical spectral behavior of the LPG fiber device described by the waveguide refractive index geometry of LPG1, showing the wavelength shift of the sixth cladding mode resonance as a function of curvature.

and $n_{\mathrm{co}}^{01}$ is the effective index of the core mode, and $n_{\mathrm{cl}}^{1 \nu}$ is the effective index of the $\nu$ th radial cladding mode; both indexes being dependent on the core refractive index $n_{1}$, the cladding refractive index $n_{2}$, and the wavelength $\lambda$. Furthermore, $n_{\mathrm{cl}}^{1 \nu}$ is a function of the refractive indexes of the surrounding medium $n_{s} . \Lambda$ is the period of the LPG, $T$ is the temperature, $\varepsilon$ is the strain experienced by the fiber, and $R$ is the curvature imposed on the fiber. The quantity $\delta n_{\mathrm{eff}_{\nu}}$ is the differential effective index between the core and the cladding modes. In (3), the superscripts denote the $L P_{01}$ core mode and the $H E_{1 \nu}$ axially symmetric cladding modes. Modeling the cladding modification used some radial refractive-index measurements of the femtosecond-laser-inscribed waveguides in bulk glass and a piecewise procedure. These measurements gave the approximate indexes and radial trends across the modification to be employed in the finite-element package. The index profile used has a central void region of index $n=1$ with a diameter of $1.6 \mu \mathrm{m}$ surrounded by a radially symmetric index perturbation (from that of silica) of $0.01 \exp [-2.2(r-0.8)]$ (where $r$ is in measured micrometers) extending to $3.5 \mu \mathrm{m}$, giving an effective cross-sectional area of $5.1 \mu \mathrm{m}$.

Using the effective refractive indexes of the core and cladding modes at various wavelengths obtained from FEM$\mathrm{LAB}$, it is possible to obtain estimates for the group refractive indexes of the cladding and the core modes. Furthermore, the effective refractive indexes of the core and the cladding modes at various curvatures (both concave and convex bending) at a given wavelength were calculated using FEMLAB. The presence of the bending was treated by using the conformal mapping technique [12], which replaces the curved optical fiber waveguide by an equivalent straight waveguide with the following index profile as a function of curvature $R$ and longitudinal strain $\varepsilon$, i.e.,

$$
n(R) \rightarrow\left(n(0, \lambda)+\frac{d n}{d \varepsilon} \cdot \varepsilon\right) \cdot \exp \left(\frac{d}{R}\right)
$$

where $n(0)$ is the initial refractive index of the core/cladding at a wavelength of $\lambda, R$ is the curvature experienced by the fiber, and $d$ is the distance from the center of the fiber. The strain information was obtained using the mechanical finite-element analysis (FEA) package and used in conjunction with the strainoptic coefficients of the core material $\left(d n_{\mathrm{co}} / d \varepsilon=2.7 \times 10^{-7}\right)$ and outer cladding material $\left(d n_{\mathrm{cl}} / d \varepsilon=2.4 \times 10^{-7}\right.$ from [13]) to obtain the index variation due to strain. The strain-optic coefficients of a glass material will vary with the composition and concentration of dopants, but for simplicity, we assumed that these coefficients are constant for each layer of the optical fiber and that the coefficients given in [13] are a close approximation to those of the fibers employed. The package was used to obtain the effective refractive indexes of the core and cladding modes and their group indexes at rotational angles of $0^{\circ}$ (a concave bend) and $180^{\circ}$ (a convex bend). The modeling of both these E-core and symmetric fibers showed that the core mode undergoes little variation with both modes of bending. This explains the blue and red wavelength shifts observed for the modified cladding of the LPG in the SMF as the majority of the strain occurs in the cladding, which is asymmetric (due to the inscribed index modification, there is an effect on the effective index of the cladding modes and thus the phase matching condition of the attenuation bands, which is then dependent upon the orientation of the bend), and thus, the magnitude and sign of the strain are dependent upon the bending orientation. The theoretical wavelength shift was obtained using the same approach as [4], giving

$$
\Delta \lambda=\left[\frac{\lambda}{\left(\delta n_{\mathrm{eff}}-\delta n_{g}\right)} \cdot \frac{d \delta n_{\mathrm{eff}}}{d R}+\frac{\left(\delta n_{\mathrm{eff}}\right)^{3}}{\delta n_{g}} \frac{d \Lambda}{d R}\right] \cdot \Delta R
$$

where $\delta n_{\text {eff }}=n_{\text {coeff }}-n_{\text {cleff }_{\nu}}$ is the differential effective index between the sixth cladding mode (as used in the experiment) and the core mode, and $\delta n_{g}=n_{\operatorname{cog}}-n_{\operatorname{clg}_{\nu}}$ is the differential group index. The effective refractive indexes of the core and the sixth cladding mode were calculated as a function of curvature from which the wavelength shift of the attenuation band was determined (see Fig. 15).

The modeling of the modified LPG in SMF produced the same general spectral behavior with respect to curvature as observed experimentally, although the spectral sensitivities of the various attenuation bands were different. In the case of modeling the spectral response to the curvature of the E-core fiber with the asymmetric cladding, the general functionality observed experimentally was also qualitatively similar, but again, there were differences between the theoretical results and experimental data. The differences are not surprising because the shape of the modified refractive-index profile of the LPG device is not known precisely.

\section{CONCLUSION}

In this paper, we have shown that by using a femtosecond laser system to modify the cladding of an SMF containing 
an LPG, it is possible to measure the curvature of the fiber and determine the direction of the bending in a plane containing the cladding modification. Compared with the previously demonstrated directional bend sensors, these devices have the advantage of being based on standard telecommunication grade fiber, which both facilitates the connection of the device into a fiber system and also results in minimal polarization dependence, thereby simplifying interrogation. Modeling the cladding modification as a change in the fiber refractive-index profile gives good qualitative agreement with the experimentally observed behavior, although further research is needed to rule out any possible contribution from asymmetric changes to the mechanical properties of the fiber.

To study the polarization properties of these devices, cladding modification was also carried out on an E-core fiber containing an LPG. In this fiber, it is possible to separate the response to the two polarization eigenstates as they lead to widely separated attenuation bands in the transmission spectrum. A cladding modification applied on the fast axis of the fiber is seen to affect the light in the fast axis more significantly than the light in the orthogonal state. Further work to optimize the cladding modification may lead to a device capable of determining the direction of a bend in two dimensions.

\section{REFERENCES}

[1] T. Erdogan, "Cladding-mode resonances in short- and long-period fibre Grating filters," J. Opt. Soc. Amer. A, Opt. Image Sci., vol. 4, no. 8, pp. 1760-1773, 1997.

[2] A. D. Kersey et al., "Fibre grating sensors," J. Lightw. Technol., vol. 15, no. 8, pp. 1442-1462, Aug. 1997.

[3] T. Allsop et al., "Embedded progressive-three layered fibre long-period gratings for respiratory monitoring," J. Biomed. Opt., vol. 8, no. 3, pp. 552-558, Jul. 2003.
[4] - "The spectral characteristics of long period gratings written in D-shaped optical fiber as a bending sensors," presented at the Optical Fiber Sensors (OFS), Nara, Japan, Oct. 2003, Paper TuP-8.

[5] H. J. Patrick, "Self-aligning, bipolar bend transducer based on long period grating written in eccentric core fibre," Electron. Lett., vol. 36, no. 21, pp. $1763-1764$, Oct. 2000.

[6] A. Martinez et al., "Point by point FBG inscription by a focused NIR femtosecond laser," presented at the OSA Conf.-Conf. Lasers and Electro-Optics/Int. Quantum Electronics Conf. (CLEO/IQEC) and Photonic Applications, Systems and Technologies (PhAST), Washington, DC, 2004, CMY6.

[7] O. Duhem et al., "Effect of UV-induced birefringence on long period-grating coupling characteristics," Electron. Lett., vol. 5, no. 36, pp. 456-417, Mar. 2000.

[8] A. M. Vengsarkar et al., "Birefringence reduction in side-written photoinduced fiber devices by a dual-exposure method," Opt. Lett., vol. 19, no. 16, p. 1260 , Aug. 1994.

[9] W. Du, H. Tam, M. Liu, and X. Tao, "Long-period grating bending sensors in laminated composite structures," in Proc. SPIE Conf-_Smart Structures and Mater. San Diego, CA: SPIE, 1998, vol. 3330, p. 284

[10] T. Allsop, F. Floreani, D. J. Webb, and I. Bennion, "The bending and temperature characteristics of long period gratings written in elliptical core step-index fibre," presented at the Optical Fiber Sensors (OFS)-17, Bruges, Belgium, 2005, Paper P2-30.

[11] T. Allsop, H. Dobb, V. Mezentsev, T. Earthgrowl, A. Gillooly, D. J. Webb, and I. Bennion, "Spectral sensitivity of long period gratings fabricated in elliptical core D-shaped optical fiber," in Proc. SPIE Opt. East, Philadelphia, PA, Oct 2004, vol. 5589, pp. 266-277.

[12] M. Heiblum and J. H. Harris, "Analysis of curved optical waveguides by conformal transformation," IEEE J. Quant. Electron., vol. QE-11, no. 2, pp. $75-83$, Feb. 1975

[13] T. Allsop et al., "A comparison and characteristics of sensing parameters of long period gratings written in three different types of fiber," Opt. Fiber Technol., vol. 9, no. 4, pp. 210-223, Oct. 2003.

Photographs and biographies of author and co-authors not available at the time of publication. 